\title{
Alterations in DNA methyltransferases and methyl-CpG binding domain proteins during cleft palate formation as induced by $2,3,7,8$-tetrachlorodibenzo- $p$-dioxin in mice
}

\author{
WEI ZHANG ${ }^{1}$, SHANSHAN ZHOU ${ }^{2}$, YUWEI GAO $^{3}$, HONGQUAN SONG $^{3}$, \\ XIAOHUI JIAO ${ }^{3}$, XIAOTONG WANG ${ }^{3}$ and YONG $\mathrm{LI}^{3}$ \\ ${ }^{1}$ Department of Stomatology, The Fourth Affiliated Hospital, Harbin Medical University; \\ Departments of ${ }^{2}$ Neurology and ${ }^{3}$ Oral and Maxillofacial Surgery, The First Affiliated Hospital, \\ Harbin Medical University, Harbin, Heilongjiang 150001, P.R. China
}

Received October 8,2017; Accepted January 23, 2018

DOI: $10.3892 / \mathrm{mmr} .2018 .8521$

\begin{abstract}
Maternal exposure to 2,3,7,8-tetrachlorodibenzo-p-dioxin (TCDD) induces cleft palate formation in mice. This TCDD treatment, which may be considered an environmental factor in cleft palate formation, is associated with alterations in DNA methylation. However, the underlying molecular mechanisms of DNA methylation produced by TCDD in mouse embryos are poorly understood. DNA methyltransferases (DNMTs) and methyl-CpG binding domain proteins (MBDs) are thought to be closely associated with the actions of DNA methylation. Therefore, the present study tested the hypothesis that this cleft palate inducing effect of TCDD will alter the expression levels of DNMTs and various MBDs in palate tissue of fetal mice. Pregnant C57BL/6J mice were treated with either TCDD $(64 \mu \mathrm{g} / \mathrm{kg})$ or corn oil (control) at embryonic day 10.5 (E10.5) and fetal palates were harvested for structural and molecular analyses at E13.5, E14.5, E15.5 and E17.5. Expression levels of DNMTs and MBDs were assayed using reverse transcription-quantitative polymerase chain reaction and western blotting. The incidence of cleft palates in the TCDD group was $98.24 \%$, whereas no cases of cleft palate were observed in the control group. Expression levels of DNMTs and MBDs were significantly increased in the TCDD group compared with the control. The results demonstrate
\end{abstract}

Correspondence to: Dr Xiaohui Jiao, Department of Oral and Maxillofacial Surgery, The First Affiliated Hospital, Harbin Medical University, 143 Yiman Street, Nangang, Harbin, Heilongjiang 150001, P.R. China

E-mail: jiaoxiaohui_dds@163.com

Abbreviations: $\mathrm{CL} / \mathrm{P}$, cleft lip with or without cleft palate; TCDD, 2,3,7,8-tetrachlorodibenzo- $p$-dioxin; DNMT, DNA methyltransferase; $\mathrm{MBD}$, methyl-CpG binding domain protein

Key words: cleft palate, DNA methyltransferase, methyl-CpG binding domain protein, 2,3,7,8-tetrachlorodibenzo- $p$-dioxin, mice clear alterations in DNMTs and MBDs, as induced by TCDD, and suggest that such alterations are important in cleft palate formation in fetal mice.

\section{Introduction}

Cleft lip with or without cleft palate $(\mathrm{CL} / \mathrm{P})$ is one of the most prevalent developmental deformities world-wide, with a prevalence ranging from approximately $1 / 300$ to $1 / 2,500$ of live births $(1,2)$. CL/P results from a complex etiology due to multiple genetic and environmental risk factors (3). Environmental factors, such as smoking, alcohol, caffeine, infections, corticosteroids, retinoic acid, dioxin, as well as other occupational pollutants, can seriously affect development of the fetus during pregnancy to eventually result in conditions such as CL/P (4).

In specific, 2,3,7,8-tetrachlorodibenzo- $p$-dioxin (TCDD), a highly toxic halogenated environmental contaminant that produces adverse biological effects, including developmental toxicity and teratogenesis (5), has been shown to result in the formation of a cleft palate within mice (6). Recent findings have established that epigenetic modifications are involved in murine palatogenesis induced by TCDD, with DNA methylation being considered as playing a key role in cleft palate formation (7). The significance of DNA methylation has been revealed from findings demonstrating that global DNA methylation status was substantially enhanced in fetal mice following maternal exposure to TCDD, and this factor was thought to be a causative factor in palate malformation (8). However, the underlying molecular mechanisms of DNA methylation caused by TCDD in mice require further elucidation.

DNA methylation is among the best studied epigenetic modifications and is closely related to disease and homeostatic imbalances in mammals, through its capacity of participating in proper embryonic development, gene expression regulation and even tumorigenesis $(9,10)$. The dynamic patterns of DNA methylation are catalyzed by DNA methyltransferases (DNMTs) and then read and interpreted by specific proteins, called methyl-CpG binding domain proteins (MBDs) (11). As it has been reported that congenital cleft palate patients usually show 
Table I. Mouse primers used for reverse transcription-quantitative polymerase chain reaction.

\begin{tabular}{lll}
\hline Primers & \multicolumn{1}{c}{ Forward $\left(5^{\prime}-3^{\prime}\right)$} & \multicolumn{1}{c}{ Reverse $\left(5^{\prime}-3^{\prime}\right)$} \\
\hline Dnmt 1 & TGGAGCCCAGCAAAGAGTA & TAATGGTAGAAGGAGGAACAGTG \\
Dnmt3a & AACGGAAACGGGATGAGTG & TCGTCGGCTGCTTTGGTAG \\
Dnmt3b & CGAAGACGCACAACCAATG & ACAGAGCCCACCCTCAAAG \\
Mecp2 & AAGCCTCTGAGACCCTATCC & GCAGTGGAGACAACCCTT \\
Mbd2 & CAGCTCCATTGCCTGCATAG 3 AGATGAATAAGAGTCGCCAG & GGAATCAGACGGAAAGAAAA \\
$\beta$-actin & AGATGAACACATCCACTTAGC \\
\hline
\end{tabular}

DNMT, DNA methyltransferases; MBD, methyl-CpG-binding domain proteins; Mecp2, methyl-CpG binding protein 2.

distinct methylation profiles (12), we hypothesized that DNMTs and MBDs, which greatly affect DNA methylation patterns, would participate in cleft palate formation induced by TCDD. It is well established that DNA methyltransferases are crucial for establishing DNA methylation patterns in mammals, and are thus responsible for maintenance of methylation (DNMT1) and de novo methylation (DNMT3a, 3b) $(13,14)$. The methyl-CpG binding domain proteins, especially MeCP2, MBD2, MBD3, are critical players in determining the transcriptional state of epigenosomes, and therefore play an important role as facilitators of epigenetic-based repression (15). Results from previous studies have demonstrated that cigarette smoke and alcohol cause changes in the expression of DNA methyltranferases and methyl-CpG binding domain proteins in murine embryonic cells $(16,17)$. In this study, we utilized the mouse cleft palate model as accomplished by maternal exposure to TCDD, to elucidate the effects of epigenetic regulation, including DNMT1, DNMT3a, DNMT3b, MeCP2, MBD2 and MBD3 expression in vivo, and investigated their relationship and participation in TCDD-induced cleft palate.

\section{Materials and methods}

Animals. The present study was approved by the Transformation Center of Harbin Medical University (Harbin, China). C57BL/6J mice (8-10 weeks of age) consisting of 30 female experimental mice and 15 male mice used to impregnate the females were purchased from the Institute of Laboratory Animals of the Chinese Academy of Medical Sciences. The mice were housed under controlled laboratory conditions of $22-24^{\circ} \mathrm{C}, 55 \pm 5 \%$ humidity, under a $12 / 12 \mathrm{~h}$ light/dark cycle, with standard laboratory chow and water ad libitum. After being acclimated to the laboratory conditions, two female mice were paired with one male overnight. Presence of vaginal plugs on the following morning was interpreted as a successful mating and was designated as embryonic day 0.5 (E0.5).

Drug administrations. At E10.5, pregnant mice were randomly divided into two groups: i) TCDD group-consisted of gavage with a single dose of $64 \mu \mathrm{g} / \mathrm{kg}$ TCDD; and ii) control group-mice that received an equal volume of corn oil. Following treatment, pregnant female mice were caged and fed separately. At E13.5, E14.5, E15.5 or E17.5, mice were euthanized using $\mathrm{CO}_{2}$. A total of three pregnant mice were euthanized at each time-point, and the total number of fetal mice present was recorded. Fetal palate tissue was quickly isolated from each embryo and stored at $-80^{\circ} \mathrm{C}$.

Histological staining of sections. For histological analysis, the heads of the fetal mice were fixed in $4 \%$ paraformaldehyde (PFA), dehydrated in a graded series of ethanol, embedded in paraffin and then sectioned at $10 \mu \mathrm{m}$ in the coronal plane. To assess the general morphology, deparaffinized sections were subjected to hematoxylin (Solarbio, Beijing, China) and eosin (Sinopharm Chemical Reagent Co., Ltd., Beijing, China) staining using established procedures.

Reverse transcription-quantitative polymerase chain reaction (RT-qPCR). Total RNA was isolated from palatal shelves using a RNeasy Mini kit (BioTeke, Beijing, China) according to the manufacturer's instructions. The Super M-MLV RT kit (BioTeke) was used to synthesize first-standed cDNA. Each q-PCR reaction system was performed in a total volume of $20 \mu \mathrm{l}$ : cDNA template $1 \mu \mathrm{l}$, forward and reverse primers $(10 \mu \mathrm{m})$ each $0.5 \mu \mathrm{l}$, SYBR-Green Mastermix $10 \mu \mathrm{l}$ and $\mathrm{ddH}_{2} \mathrm{O}$ $8 \mu \mathrm{l}$. The amplification protocol consisted of 40 cycles of denaturation for $10 \mathrm{sec}$ at $95^{\circ} \mathrm{C}$, annealing for $20 \mathrm{sec}$ at $60^{\circ} \mathrm{C}$, and elongation for $30 \mathrm{sec}$ at $72^{\circ} \mathrm{C}$.

A SYBR-Green fluorescence quantification system (Bioneer, Daejeon, Korea) was utilized to perform the Real-time PCR analysis. $\beta$-actin (Santa Cruz Biotechnology, Inc., Santa Cruz, CA, USA) was used as an internal control and gene-specific mRNA expression was normalized to $\beta$-actin expression by using the $2^{-\Delta \Delta C t}$ method. Primer sequences are shown in Table I.

Western blotting. Proteins extracts of the TCDD and control groups from E13.5 to E15.5 were performed using standard protocols and antibodies recognizing DNMT1, DNMT3a, DNMT3b, MeCP2, MBD2 and MBD3 (all from Bioss, Beijing, China). All data were normalized to the expression of $\beta$-actin (Santa Cruz Biotechnology, Inc.). The intensity of the bands was analyzed by densitometry.

Statistical analysis. Data from E13.5 to E15.5 in the two groups were presented as the mean \pm SD and analyzed using SPSS 17.0 (SPSS, Inc., Chicago, IL, USA). Independent t-tests were used to establish statistically significant differences 

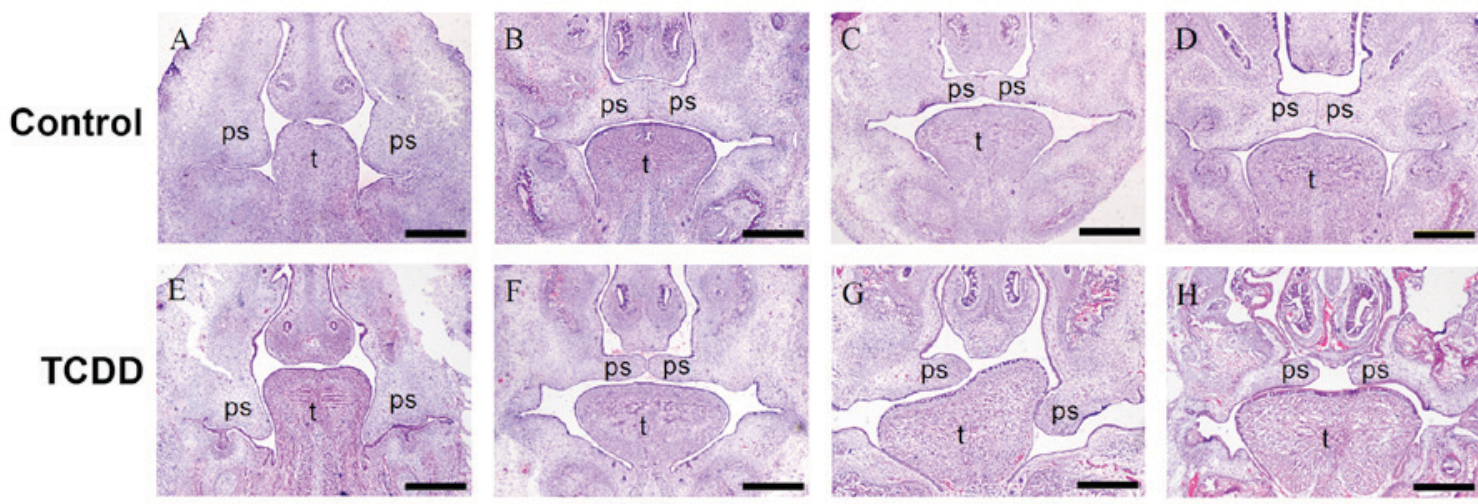

E13.5

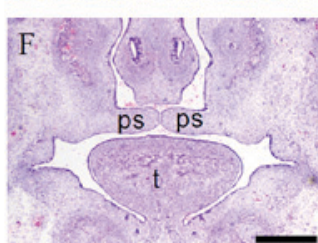

E14.5

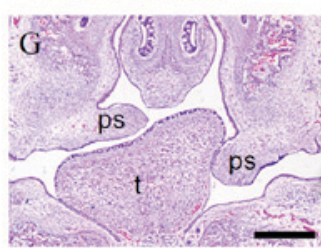

E15.5

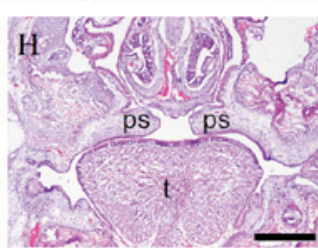

E17.5

Figure 1. Histology of the palate. (A-D) H\&E stained coronal sections showing normal palatogenesis in control group at E13.5, E14.5, E15.5 and E17.5 in mice. (E-H) Coronal sections of TCDD group at E13.5, E14.5, E15.5 and E17.5, showing a failure of palate shelf elevation in mice. PS, palatal shelf; T, tongue; Scale bars, $500 \mu \mathrm{m}$.

A

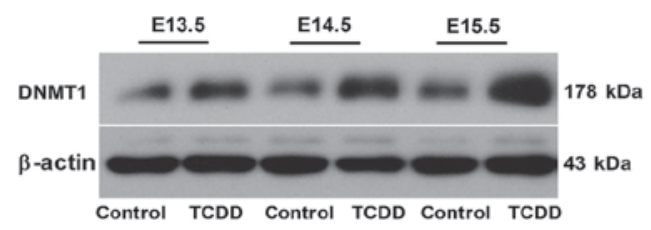

D

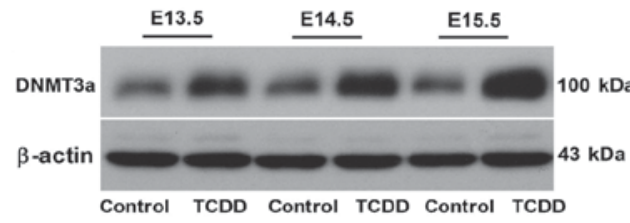

G

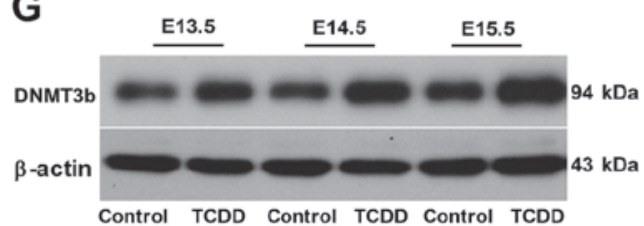

B

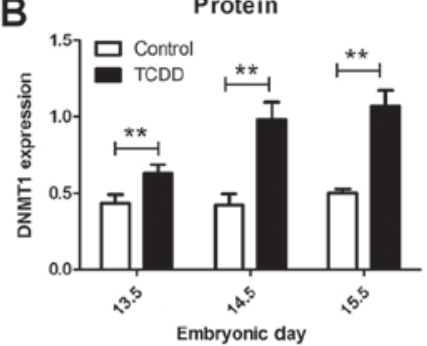

E
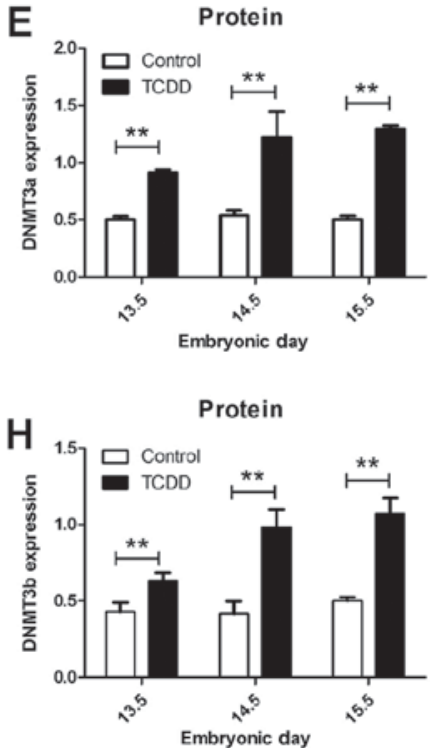

C

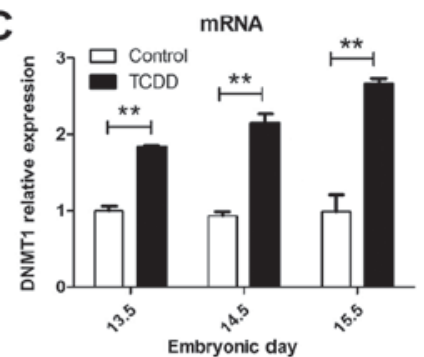

F
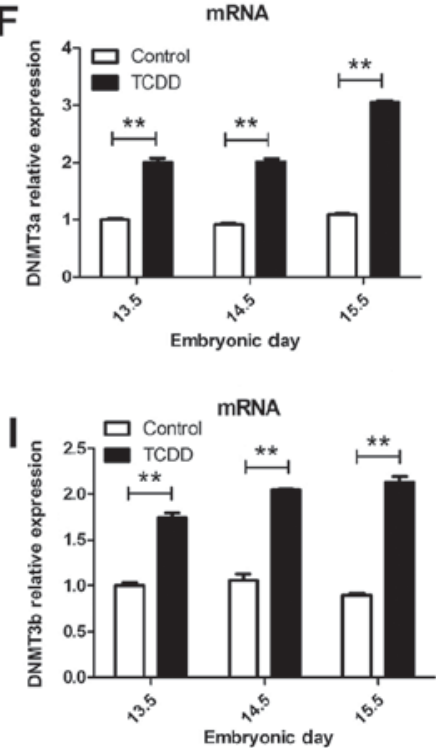

Figure 2. Induction of DNMTs in palatal shelves by TCDD. (A-C) Protein and mRNA expression levels of DNMT1 in palatal shelves at E13.5, E14.5 and E15.5 by TCDD and corn oil as control. Values were normalized against expression of $\beta$-actin. Error bars represent SD. ${ }^{* *} \mathrm{P}<0.01$. (D-F) DNMT3a protein and mRNA levels in palatal shelves by TCDD and corn oil control at E13.5, E14.5 and E15.5. (G-I) DNMT3b protein and mRNA levels in palatal shelves by TCDD and corn oil control at E13.5, E14.5 and E15.5. DNMTs, DNA methyltransferases.

between two groups. A $\mathrm{P}<0.05$ was required for results to be considered statistically significant. All experiments were performed at least in triplicate.

\section{Results}

TCDD treatments at E10.5 induced cleft palate in mice. In fetuses excised from pregnant females who received TCDD at E10.5, the number of fetuses with $\mathrm{CP}$ only, without other obvious orofacial defects were: 14/14 at E13.5, 16/16 at E14.5, $13 / 14$ at E15.5, and 13/13 at E17.5. In contrast, no cleft palates were observed in fetal mice within the control group.

The morphological features of palatal development from E13.5 to E17.5 in fetuses from pregnant females treated with TCDD were examined under a stereomicroscope (Fig. 1). $\mathrm{H} \& \mathrm{E}$ staining revealed that at E13.5, bilateral palate shelves of 
A

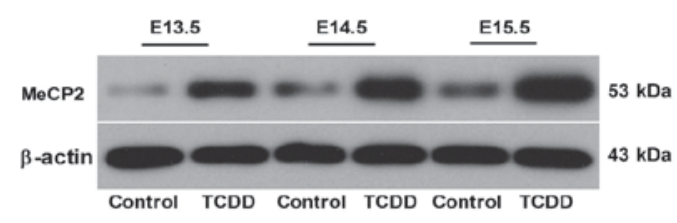

D

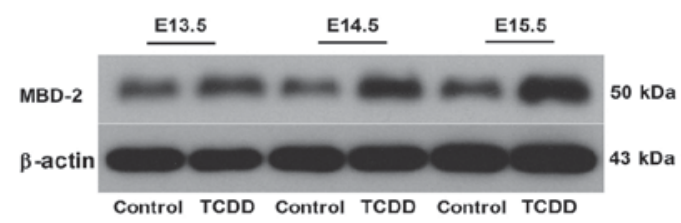

G

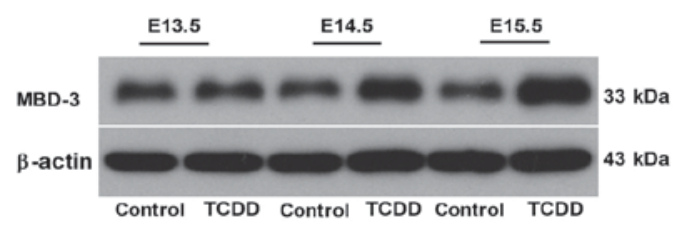

B

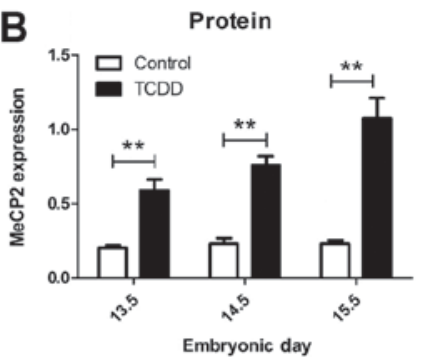

E
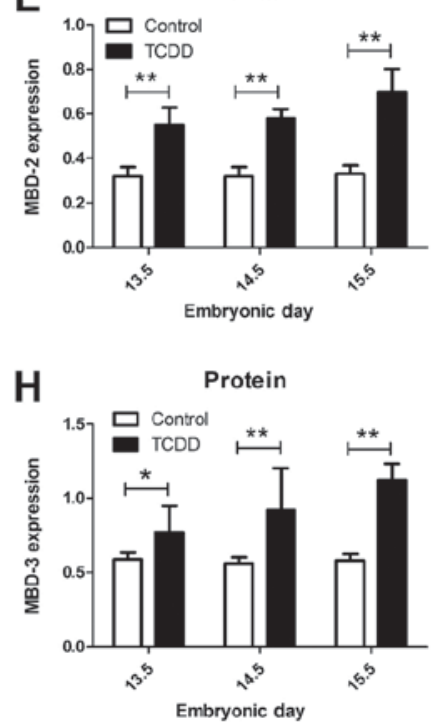

C
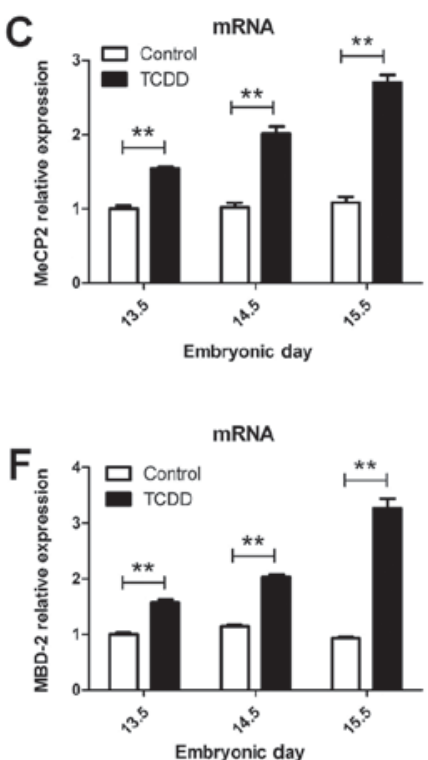

I

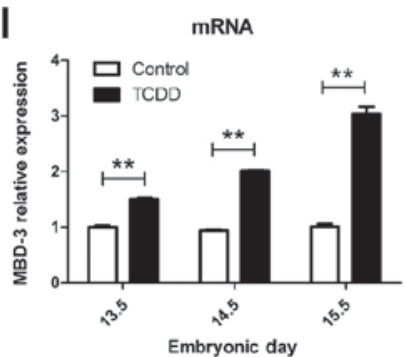

Figure 3. Effect of TCDD on protein and mRNA expression levels of MBDs. (A-C) The protein expression levels of MeCP2 in palatal shelves by TCDD and corn oil control at E13.5, E14.5 and E15.5 were detected by western blotting. The mRNA levels of MeCP2 were examined by real-time quantitative PCR. Values were normalized against expression of $\beta$-actin. Error bars represent $\mathrm{SD} .{ }^{*} \mathrm{P}<0.05$ and ${ }^{* * *} \mathrm{P}<0.01$. (D-F) MBD2 protein and mRNA levels in palatal shelves by TCDD and corn oil control at E13.5, E14.5 and E15.5. (G-I) MBD3 protein and mRNA levels in palatal shelves by TCDD and corn oil control at E13.5, E14.5 and E15.5. MBDs, methyl-CpG binding domain proteins.

TCDD mice were observed to project downward on each side of the tongue and failed to elevate, which was similar to that observed in controls. At E14.5, palates of the controls were in the fusion stage, while TCDD-treated embryos showed bilateral palate shelves that were not fused. At E15.5 and E17.5, fetuses in the control group demonstrated a complete palate; while the absence of a successful adherence in palate shelves within the TCDD group resulted in a cleft palate.

TCDD activated the expression of DNMTs in palatal shelves. To identify which DNA methyltransferase was regulated, we performed real-time quantitative PCR and western blot analysis for DNMT1, DNMT3a and DNMT3b. $\beta$-actin served as an internal reference and relative transcript and protein levels are shown in Fig. 2. Our results revealed that mRNA levels of DNMT1, DNMT3a and DNMT3b were significantly increased in the TCDD, as compared with that of the control group at E13.5 to E15.5 ( $\mathrm{P}<0.01)$. Within palatal shelves, a strong expression in DNMTs was obtained in the TCDD group from E13.5 to E15.5, while a relatively weak expression was present in the control group.

MeCP2, MBD2 and MBD3 are upregulated by TCDD in mice palates. The mRNA expression of methyl-CpG binding domain proteins was detected using real-time quantitative PCR. The
mRNA levels of MeCP2, MBD2 and MBD3 in the TCDD group at E13.5 to E15.5 were significantly increased $(\mathrm{P}<0.05)$, compared with those of controls (Fig. 3). Protein expressions of MeCP2, MBD2 and MBD3 were identified using western blotting. Results from protein imprinting showed that protein expression levels of the TCDD group were significantly greater than in controls from E13.5 to E15.5 $(\mathrm{P}<0.05)$, which was in accordance with the trend observed for mRNA.

Temporal accumulations of DNMTs and MBDs in mouse embryonic palates. The intensity of the bands resulting from western blot analysis was analyzed by densitometry. At E13.5, positive staining was sparsely present in palatal shelves. At E14.5, DNMTs and MBDs were strongly expressed in palatal shelves, with the strongest expression of DNMTs and MBDs being detected at E15.5 within the TCDD group.

\section{Discussion}

$\mathrm{CL} / \mathrm{P}$ is a common birth defect notable for its significant morbidity and complex etiology. The complex etiology reflects the interaction of multiple genetic, epigenetic and environmental factors. One potential basis for this interaction involves that of environmental factors which can then affect gene expression via alterations in DNA methylation epigenetic 
modifications. DNA methylation is well known as an epigenetic marker involved in numerous biological processes which can induce gene expression changes without altering DNA sequences (18). Although Kuriyama et al (19) have reported that DNA methylation may be crucial for murine palatogenesis, the precise molecular underpinnings directing epigenetic alterations that result in cleft palate remain obscure.

In eukaryotic cells, the establishment and maintenance of methylation patterns depends on the methylation modification system, which involves two important proteins, DNMTs and MBDs. Associated with this process are three enzymatically active DNA methaltransferases (DNMT1, DNMT3a, DNMT3b). DNMT1 is a maintenance methyltransferase that ensures inheritance of methylation patterns during cell division by preferentially methylating hemimethylated $\mathrm{CG}$ dimucleotides. DNMT3a and DNMT3b are responsible for de novo methylation, establishing DNA methylation patterns in germs cells. MeCP2, MBD2 and MBD3 are members of the MBD family of proteins that are associated with transcriptional activation or repression, regulation of chromatin structure, pluripotency, development and differentiation (20). Mukhopadhyay et al (16) demonstrated that upon exposure to $80 \mu \mathrm{g} / \mathrm{ml}$ cigarette smoke extract for $24 \mathrm{~h}$, first branchial arch-derived (1-BA) cells, which contribute to cleft palate formation, exhibited a decline in global DNA methylation and triggered a proteasomal-mediated degradation of DNMTs (DNMT1, DNMT3a), MeCP2 and MBD3. However, as this study was performed using an in vitro preparation, the question of whether such changes in gene expression of DNMTs and MBDs would be present in vivo has yet to be determined.

TCDD is a teratogenic reagent shown to be effective in inducing cleft palate in mice and therefore has been widely used as a model for the study of palate formation. Gestational exposure to TCDD can be considered as an environmental factor which influences the molecular development of palate tissue. Accordingly, we utilized this cleft palate model and directed our attention at examining potential in vivo changes that might be present in the expressions of DNMTs and MBDs in this model.

In this study, exposure of pregnant C57BL/6J mice to TCDD was effective in producing cleft palate in the fetuses. With use of a TCDD dose of $64 \mu \mathrm{g} / \mathrm{kg}$ as administered at E10.5, we obtained a $98.24 \%$ incidence of cleft palate. No other apparent external anomalies or complications were observed in the craniofacial tissue of these TCDD exposed litters. The morphological outcomes in terms of palatal development at E13.5, E14.5, E15.5 fetuses were evaluated with use of a stereomicroscope. H\&E staining of embryo head sections at E14.5 and E15.5 showed a failure of palatal fusion in the TCDD group resulting in a cleft defect. By contrast, palatal shelve fusion was complete in the control group.

As it has been reported that abnormal methylation status may be a probable reason for cleft palate $(12,19,21)$, we next examined DNMT gene expression patterns in these mice. Our findings reveal that developmental exposure to TCDD significantly affects DNMT gene expression patterns. Results obtained from q-PCR analysis showed that Dnmt1, Dnmt3a and Dnmt3b were overexpressed at E13.5 in the TCDD group, and increased further at later stages of development. This trend was consistent with results obtained from western blotting. Overall, these changes are in accordance with recent studies by Wang et al (8). One slight difference between our current results and that of Wang et al was the observation of a decline in the expression of Dnmt3b in their group, a difference which may be attributable to their use of a lower dose of $28 \mu \mathrm{g} / \mathrm{kg}$ TCDD as administered via oral gavage in the mice. However, the increased expression of DNMTs was clearly suggestive of stimulation by TCDD in maintenance (DNMT1) and de novo (DNMT3a and DNMT3b) DNMT activities. The fact that DNA methyltransferase activity is considered to be increased by folic acid (22), and folic acid is regarded as the most effective treatment for fetal congenital malformations, including neural tube defects and CL/P $(22,23)$, the significance of these increased expressions of DNMTs can be appreciated. Interestingly, the lack of folic acid intake in the peri-conceptional period has been identified as one of the high risk factors for CL/P (24). Accordingly, we hypothesized that the aberrant increase of DNMTs may consume a large amount of folic acid and result in a folate deficiency within the body.

Coinciding with the changes in the expression of DNMT genes, we also observed a significant increase in MBDs during palatal development. Specifically, we observed that TCDD produced a significant over expression in both mRNA and proteins levels of the three methyl-CpG binding domain proteins, MeCP2, MBD2 and MBD3, in the palatal shelves of mice fetuses. These findings differ from those of Aluru et al (14) who reported a degradation in the expressions of MeCP2, MBD2 and MBD3 in zebrafish, the difference is likely due to the different species of mice and zebrafish. With regard to MBDs, Zhang et al (25) reported that MeCP2 was indispensable for normal neurological functioning and over expression of MeCP2 in mice resulted in neurobehavioural disorders, dendritic abnormalities and synaptic defects. Hendrich et al (26) showed that MBD2 and MBD3 played selective roles with regard to their function as transcriptional repressors. While $\mathrm{Mbd}^{-/-}$mice died during early embryogenesis, Mbd2 $2^{--}$mice were viable and fertile, suggesting that MBD2 and MBD3 exert distinct but interacting effects in mouse development. The results presented here are in accord with the changes in DNMTs expression levels observed, and it seems likely that the combined actions of DNMTs and MBDs may ultimately contribute to the aberrant methylation status of mice as induced by TCDD. The differential recruitment of DNA methyltransferases and methyl CpG-binding domain proteins are considered as factors which initiate the repression and silencing of Oct 4 via hypomethylation of the Oct4 promoter (27). As based upon a review of a large genome-wide association study of datasets, Oct4 was deemed to represent a loci harbor gene associated with CL/P (28).

In conclusion, the data presented here, which show increased recruitment of DNMTs and MBDs, and TCDD effects on DNMTs and MBDs expression as demonstrated in vivo in this mouse model of cleft palate, suggest that both the establishment and maintenance of methylation patterns may play an important role in murine palatogenesis.

\section{Acknowledgements}

This study was revised for submission in English by the ED-IT Editorial Service. 


\section{References}

1. Murray JC: Gene/environment causes of cleft lip and/or palate. Clin Genet 61: 248-256, 2002.

2. Stanier P and Moore GE: Genetics of cleft lip and palate: Syndromic genes contribute to the incidence of non-syndromic clefts. Hum Mol Genet 1: R73-R81, 2004.

3. Adeyemo WL and Butali A: Genetics and genomics etiology of nonsyndromic orofacial clefts. Mol Genet Genomic Med 5: 3-7, 2017.

4. Honein MA, Rasmussen SA, Reefhuis J, Romitti PA, Lammer EJ, Sun L and Correa A: Maternal smoking and environmental tobacco smoke exposure and the risk of orofacial clefts. Epidemiology 18: 226-233, 2007.

5. Abbott BD: Review of the interaction between TCDD and glucocorticoids in embryonic palate. Toxicology 105: 365-373, 1995.

6. Yamada T, Hirata A, Sasabe E, Yoshimura T, Ohno S, Kitamura N and Yamamoto T: TCDD disrupts posterior palatogenesis and causes cleft palate. J Craniomaxillofac Surg 42: 1-6, 2014.

7. Yuan X, Qiu L, Pu Y, Liu C, Zhang X, Wang C, Pu W and Fu Y: Histone acetylation is involved in TCDD-induced cleft palate formation in fetal mice. Mol Med Rep 14: 1139-1145, 2016.

8. Wang C, Yuan XG, Liu CP, Zhai SN, Zhang DW and Fu YX: Preliminary research on DNA methylation changes during murine palatogenesis induced by TCDD. J Craniomaxillofac Surg 45: 678-684, 2017

9. Jones PA and Laird PW: Cancer epigenetics comes of age. Nat Genet 21: 163-167, 1999.

10. Smith ZD and Meissner A: DNA methylation: Roles in mammalian development. Nat Rev Genet 14: 204-220, 2013

11. Gigek CO, Chen ES and Smith MA: Methyl-CpG-binding protein (MBD) family: Epigenomic read-outs functions and roles in tumorigenesis and psychiatric diseases. J Cell Biochem 117: 29-38, 2016.

12. Sharp GC, Ho K, Davies A, Stergiakouli E, Humphries K, McArdle W, Sandy J, Davey Smith G, Lewis SJ and Relton CL: Distinct DNA methylation profiles in subtypes of orofacial cleft. Clin Epigenetics 9: 63, 2017.

13. Jurkowska RZ, Jurkowski TP and Jeltsch A: Structure and function of mammalian DNA methyltransferases. Chembiochem 12 206-222, 2011.

14. Aluru N, Kuo E, Helfrich LW, Karchner SI, Linney EA, Pais JE and Franks DG: Developmental exposure to 2,3,7,8-tetrachlorodibenzo-p-dioxin alters DNA methyltransferase (dnmt) expression in zebrafish (Danio rerio). Toxicol Appl Pharmacol 284: 142-151, 2015.

15. Du Q, Luu PL, Stirzaker C and Clark SJ: Methyl-CpG-binding domain proteins: Readers of the epigenome. Epigenomics 7: 1051-1073, 2015
16. Mukhopadhyay P, Rezzoug F, Kaikaus J, Greene RM and Pisano MM: Alcohol modulates expression of DNA methyltranferases and methyl $\mathrm{CpG}-/ \mathrm{CpG}$ domain-binding proteins in murine embryonic fibroblasts. Reprod Toxicol 37: 40-48, 2013.

17. Mukhopadhyay P, Greene RM and Pisano MM: Cigarette smoke induces proteasomal-mediated degradation of DNA methyltransferases and methyl $\mathrm{CpG}-/ \mathrm{CpG}$ domain-binding proteins in embryonic orofacial cells. Reprod Toxicol 58: 140-148, 2015.

18. Seelan RS, Mukhopadhyay P, Warner DR, Webb CL, Pisano M and Greene RM: Epigenetic regulation of Sox4 during palate development. Epigenomics 5: 131-146, 2013.

19. Kuriyama M, Udagawa A, Yoshimoto S, Ichinose M, Sato K, Yamazaki K, Matsuno Y, Shiota K and Mori C: DNA methylation changes during cleft palate formation induced by retinoic acid in mice. Cleft Palate Craniofac J 45: 545-551, 2008.

20. Menafra R and Stunnenberg HG: MBD2 and MBD3: Elusive functions and mechanisms. Front Genet 5: 428, 2014.

21. Alvizi L, Ke X, Brito LA, Seselgyte R, Moore GE, Stanier P and Passos-Bueno MR: Differential methylation is associated with non-syndromic cleft lip and palate and contributes to penetrance effects. Sci Rep 7: 2441, 2017.

22. Luo S, Zhang X, Yu M, Yan H, Liu H, Wilson JX and Huang G: Folic acid acts through DNA methyltransferases to induce the differentiation of neural stem cells into neurons. Cell Biochem Biophys 66: 559-566, 2013.

23. Wehby GL and Murray JC: Folic acid and orofacial clefts: A review of the evidence. Oral Dis 16: 11-19, 2010.

24. Figueiredo RF, Figueiredo N, Feguri A, Bieski I, Mello R, Espinosa M and Damazo AS: The role of the folic acid to the prevention of orofacial cleft: An epidemiological study. Oral Dis 21: $240-247,2015$.

25. Zhang D, Yu B, Liu J, Jiang W, Xie T, Zhang R, Tong D, Qiu Z and Yao H: Altered visual cortical processing in a mouse model of MECP2 duplication syndrome. Sci Rep 7: 6468, 2017.

26. Hendrich B, Guy J, Ramsahoye B, Wilson VA and Bird A: Closely related proteins MBD2 and MBD3 play distinctive but interacting roles in mouse development. Genes Dev 15: 710-723, 2001.

27. Gu P, Xu X, Le Menuet D, Chung AC and Cooney AJ: Differential recruitment of methyl $\mathrm{CpG}$-binding domain factors and DNA methyltransferases by the orphan receptor germ cell nuclear factor initiates the repression and silencing of Oct 4 . Stem Cells 29: 1041-1051, 2011.

28. Dunkhase E, Ludwig KU, Knapp M, Skibola CF, Figueiredo JC, Hosking FJ, Ellinghaus E, Landi MT, Ma H, Nakagawa $\mathrm{H}$, et al: Nonsyndromic cleft lip with or without cleft palate and cancer: Evaluation of a possible common genetic background through the analysis of GWAS data. Genom Data 10: 22-29, 2016. 